\title{
Soluble (pro)renin receptor increased by hypoxia maintains oxidative metabolism in trophoblasts
}

\author{
Chikahito Suda1', Junichi Yatabe', Midori Yatabe1, Miki Yarita ${ }^{1,2}$ and Atsuhiro Ichihara1 \\ 1Department of Medicine II, Endocrinology and Hypertension, Tokyo Women's Medical University, Tokyo, Japan \\ 2Division of Nephrology and Hypertension, Department of Internal Medicine, The Jikei University School of Medicine, Tokyo, Japan
}

Correspondence should be addressed to J Yatabe: yatabe.junichi@twmu.ac.jp

\begin{abstract}
Elevated soluble (pro)renin receptor ( $(\mathrm{P}) \mathrm{RR})$ concentration in maternal blood is associated with gestational hypertension and preeclampsia. Placenta has abundant expression of $(\mathrm{P}) \mathrm{RR}$, and the binding of (P)RR with pyruvate dehydrogenase E1 beta subunit (PDHB) is reported to maintain oxidative metabolism. Thus, we hypothesized that placental hypoxia may increase (P)RR, and the increased (P)RR may preserve PDHB expression. Expression and functional analyses were performed using human placental trophoblast cells, mainly JAR cells. (P)RR co-immunoprecipitated and showed co-immunofluorescence with PDHB mainly in the mitochondria. Hypoxia treatment significantly increased intracellular s(P)RR protein expression, but secreted s(P)RR in the culture medium was decreased by hypoxia. Hypoxia treatment did not alter PDHB expression or activity in the basal condition, but when (P)RR was knocked down by siRNA, PDHB protein and activity were reduced by hypoxia. Acetyl-CoA, the product of PDH activity, was significantly reduced by hypoxia treatment with (P)RR siRNA. S(P)RR is generated from full-length PRR when cleaved by specific proteases. Protease inhibitor experiments suggested furin and site 1 protease as the enzymes generating s(P)RR in JAR cells, and only when treated by site 1 protease inhibitor, PF429242, PDHB protein showed a significant trend to decrease with hypoxia. In JAR cells, hypoxia increased intracellular $\mathrm{s}(\mathrm{P}) \mathrm{RR}$, and (P)RR preserved the expression and function of PDHB during hypoxia. (P)RR may help maintain oxidative metabolism and efficient energy production during placental ischemia in hypertensive disorders of pregnancy.
\end{abstract}

\author{
Key Words \\ - placenta \\ - preeclampsia \\ - ischemia \\ - renin-angiotensin- \\ aldosterone system
}

\section{Introduction}

The (pro)renin receptor ((P)RR), the product of ATP6AP2 gene, has multiple functions (Ichihara et al. 2010). Initially, (P)RR was considered to function primarily as an enhancer of tissue renin-angiotensin system (RAS) by (P)RR binding to its ligands, prorenin and renin, to stimulate intracellular tyrosine phosphorylation- dependent pathways in an angiotensin II-independent manner (Nguyen et al. 2002, Ichihara \& Yatabe 2019). The binding of prorenin/renin occurs at the N-terminal domain of (P)RR. Additional studies found that (P)RR can be a component of Wnt receptor complex as well as an accessory protein of vacuolar $\mathrm{H}^{+}$-ATPase. 
Full-length (P)RR [fl(P)RR] is cleaved by furin, ADAM19 (Kinouchi et al. 2013), and site 1 protease (Nakagawa et al. 2017) to generate soluble (pro)renin receptor $(\mathrm{s}(\mathrm{P}) \mathrm{RR})$ at the $\mathrm{N}$-terminal extracellular domain. $\mathrm{S}(\mathrm{P}) \mathrm{RR}$ can be measured in peripheral blood, and elevated $s(\mathrm{P}) \mathrm{RR}$ concentration is associated with various diseases, such as chronic kidney disease (Fukushima et al. 2013, Hamada et al. 2013, Morimoto et al. 2014), development of peripheral artery disease (Amari et al. 2016), and obesity (Kimura et al. 2016). While these association studies suggest a possible usefulness of $s(\mathrm{P})$ $R R$ as a disease marker, specific function of $s(P) R R$ had not been elucidated until a recent study showed that $s(\mathrm{P})$ RR increases aquaporin 2 expression in collecting duct cells via frizzled class receptor 8 -dependent $\beta$-catenin signaling (Lu et al. 2016).

Our group reported that high circulating s(P)RR concentration in maternal blood at early pregnancy predicted subsequent blood pressure elevation (Watanabe et al. 2012), and high plasma s(P)RR at delivery was associated with preeclampsia (Narita et al. 2016). Meanwhile, higher $s(\mathrm{P}) \mathrm{RR}$ concentration in cord blood was associated with reduced risk of fetus being small for gestational age (SGA) (Watanabe et al. 2013). (P)RR is widely expressed in various organs such as the brain, heart, liver, and kidney, and a significant amount of (P)RR is expressed also in the placenta (Nguyen et al. 2002). Placental expression of (P)RR was reported to be significantly higher in preeclamptic women than in women with normal pregnancy (Narita et al. 2016). These findings suggest a significant role of (P)RR in the placenta and preeclampsia, but at present, the function of placental (P)RR is not clear.

In the retina, the interaction between $(\mathrm{P}) \mathrm{RR}$ and pyruvate dehydrogenase E1 $\beta$ subunit (PDHB) preserves pyruvate dehydrogenase (PDH) activity (Kanda et al. 2015). Pyruvate dehydrogenase is an enzyme that links glycolysis to citric acid cycle, and if there is a similar mechanism in the placenta, the interaction of (P)RR with PDHB may support efficient energy production but may increase oxidative stress via mitochondrial aerobic respiration at the same time. We hypothesized that such phenomenon may be involved the relationship between (P)RR, preeclampsia, and fetal growth, (P)RR being protective for the fetus but damaging for the mother.

In order to test this hypothesis, we aimed to determine whether (P)RR co-expressed and interacted with PDHB in placental trophoblast cells and explored the effects of hypoxia on (P)RR and PDH.

\section{Materials and methods}

\section{Cell culture}

Human choriocarcinoma cells (JAR and JEG-3 cell) were purchased from American Type Culture Collection. JAR and JEG-3 are epithelial trophoblastic cell lines commonly used to study placental development. JAR cells were cultured in RPMI-1640 medium (Wako Pure Chemical Industries, Osaka, Japan) supplemented with $10 \%$ fetal bovine serum (Biowest, Nuaillé, France), and JEG-3 cells were cultured in Eagle's Minimum Essential Medium (Wako) supplemented with 10\% fetal bovine serum at $37^{\circ} \mathrm{C}$ and $5 \% \mathrm{CO}_{2}$. Approximately $3 \times 10^{5}$ cells were plated per $60 \mathrm{~mm}$ dish and used at 80-90\% confluence.

\section{Hypoxic treatment}

Low-oxygen concentration gas $\left(1 \% \mathrm{O}_{2}, 5 \% \mathrm{CO}_{2}\right.$, and $\left.94 \% \mathrm{~N}_{2}\right)$ or normal-oxygen gas $\left(20 \% \mathrm{O}_{2}, 5 \% \mathrm{CO}_{2}\right.$, and $75 \%$ room air) was made using a gas mixture device (LogMix; Fronto, Tokyo, Japan). Cells were exposed to hypoxic or normoxic gas in a modular incubator chamber (Billups-Rothenberg Inc., Del Mar, CA, USA) filled with mixed gas. For the experiments examining chemical hypoxia, cobalt chloride hexahydrate (Nacalai tesque, Kyoto, Japan) was dissolved in water and added to the media to achieve the final concentrations, and the cells were treated for the durations specified in the legend.

\section{Total RNA extraction and quantitative RT-PCR}

Total RNA was isolated from cultured cells using TRIzol reagent (Invitrogen) according to the manufacturer's instructions. cDNA was synthesized using High-Capacity cDNA RT Kit (Thermo Fisher Scientific Inc.) according to the manufacturer's instructions. The synthesized cDNA was used for PCR analysis using Taqman Fast Advanced Master Mix (Thermo Fisher Scientific Inc.) with the StepOnePlus real-Time PCR system (Thermo Fisher Scientific Inc.). The primers and TaqMan probe sets (TaqMan Gene Expression Assays, Inventoried) for Atp6ap2 ((P)RR) probe (Hs00997145_m1), Furin probe (Hs00965485_g1), ADAM19 probe (Hs00224960_m1), and ribosomal protein S13 (RPS13) probe (Hs01011487_g1) were purchased from Applied Biosystems (sequences not disclosed). Expression was normalized to a housekeeping gene RPS13. 


\section{Preparation of cell lysate and Western blot analysis}

Whole cell lysates were prepared using lysis buffer (Tris-buffered saline (TBS; $25 \mathrm{mM}$ Tris, $137 \mathrm{mM} \mathrm{NaCl}$, $2.68 \mathrm{mM} \mathrm{KCl}$ ), 1\% Nonidet P-40, $1 \mathrm{mM}$ DTT, protease inhibitor cocktail (Nakaraitesuku, Kyoto, Japan)). Protein samples $(10 \mu \mathrm{g})$ were separated by $12.5 \%$ SDSpolyacryamide gel electrophoresis (SDS-PAGE) and transferred to polyvinylidene difluoride membranes (GE Healthcare). Membranes were blocked in 5\% (w/v) nonfat dry milk in TBS containing $0.1 \%$ Tween 20 (TBST) at room temperature for $1 \mathrm{~h}$, followed by overnight incubation at $4^{\circ} \mathrm{C}$ with primary antibodies. Blots were subsequently washed in TBS-T and incubated for $1 \mathrm{~h}$ with the appropriate secondary IgG HRP-linked antibodies and developed with chemiluminescence reagent (Nakaraitesuku) and visualized with LuminoGraph (ATTO, Tokyo, Japan). Antibodies used were anti-ATP6AP2 ((P)RR) (Atlas Antibodies, Stockholm, Sweden, at 1:3000 dilution), anti-PDHE1- $\beta$ (PDHB) (Abcam plc, at 1:6000 dilution), anti-phosphorylated-tyrosine (p-tyr) (Santa Cruz Biotechnology, at 1:500 dilution), anti- $\beta$-Actin (Cell Signaling Technology, at 1:8000 dilution), anti-Rabbit IgG (Sigma, at 1:8000 dilution) and anti-mouse IgG (GE Healthcare UK Ltd, at 1:8000 dilution). Densitometry was performed using ImageJ software. Beta actin was used as an internal control, and for graphical representation of the Western blots, the average density ratio of target protein over beta actin of control samples was calculated and set as a reference unit for the whole membrane.

\section{Immunoprecipitation}

Whole cell lysates were prepared using lysis buffer for immunoprecipitation (1\% Nonidet P-40 in TBS with protease inhibitor cocktail). Anti-ATP6AP2 ((P)RR) (Atlas Antibodies, at 1:100 dilution) or anti-PDHB (Abcam, at 1:100 dilution) was added to the cell lysate, followed by the addition of Dynabeads Protein G (Dynal ASA, Oslo, Norway). The beads were washed three times, and bound proteins were separated by SDS-PAGE and analyzed by immunoblot with anti-ATP6AP2 ((P)RR) (Atlas Antibodies), anti-PDHB (Abcam), or anti-p-tyr (Santa Cruz Biotechnology).

\section{Immunofluorescence}

Cells were seeded in chamber slides (Matsunami Glass Industries, Osaka, Japan), washed twice with PBS before fixation with $4 \%$ paraformaldehyde phosphate buffer solution (Wako) for $15 \mathrm{~min}$ at room temperature, permeabilized with $0.1 \%$ Triton X-100, and blocked using blocking solution (Nakaraitesuku). Fixed cells were probed with the following primary antibodies: rabbit antiATP6AP2 (Atlas Antibodies, at 1:100 dilution), mouse antiPDHB (Sigma, at 1:100 dilution). Respective normal IgG (Cell Signaling Technology) at the same concentration as the primary antibody was used as negative control. The secondary antibodies used were Donkey anti-rabbit IgG Alexa Fluor 594 at 1:250 dilution and Goat anti-mouse IgG Alexa Fluor 488 (Thermo Fisher Scientific, at 1:200 dilution). MitoTracker ${ }^{\mathrm{TM}}$ Red CMXRos (Invitrogen) was used as mitochondrial marker. DAPI was used for nuclear staining, and samples were mounted using ProLong Diamond antifade reagent (Thermo). The images were acquired using a confocal laser scanning microscope (LSM-710; Carl Zeiss).

\section{Measurement of secreted s(P)RR}

$\mathrm{S}(\mathrm{P}) \mathrm{RR}$ concentration in the media was measured using Soluble (Pro)renin Receptor Assay Kit (IBL, Gunma, Japan) according to the manufacturer's protocol. The JAR cells were incubated for $24 \mathrm{~h}$ at different oxygen conditions, and the collected media were stored at $-20^{\circ} \mathrm{C}$ until the time of assay. The media were diluted 20x using the dilution buffer in the kit and measured using the ELISA method. The results were corrected for protein concentration, measured using Pierce BCA Protein Assay (Thermo Scientific, \#23227), and expressed as $\mathrm{ng} / \mathrm{mg}$ protein.

\section{siRNA treatment and ( $P$ )RR processing enzyme inhibitors}

siRNA duplexes against human ATP6AP2 and negative control (Stealth RNAi Negative Control Duplexes Medium GC Duplex \#2) were purchased from Invitrogen and used at the same concentrations. Transient knockdown of ATP6AP2 was carried out with 50 nM Stealth RNAi siRNA by Lipofectamine RNAiMAX reagent (Thermo Fisher Scientific Inc.) according to the manufacturer's instructions. Quantitative RT-PCR and Western blot analyses were performed $72 \mathrm{~h}$ after transfection with 24-h hypoxic treatment with/ without inhibitors. Furin inhibitor II and PF429242 were purchased from Sigma, and GM6001 was from Calbiochem. The vehicle controls were 1\% (v/v) DMSO 
in media for furin inhibitor II and GM6001, and 1\% (v/v) water in media for PF429242.

\section{Measurements of acetyl-CoA, pyruvate, and pyruvate dehydrogenase (PDH) activity}

PicoProbe Acetyl-CoA assay kit (BioVision, Milpitas, CA, USA), Pyruvate Dehydrogenase Activity Colorimetric Assay Kit (BioVision), and pyruvate assay kit (Abcam) were used to measure intracellular acetyl-CoA, $\mathrm{PDH}$ activity, and pyruvate concentrations, respectively. The data obtained was corrected for total protein
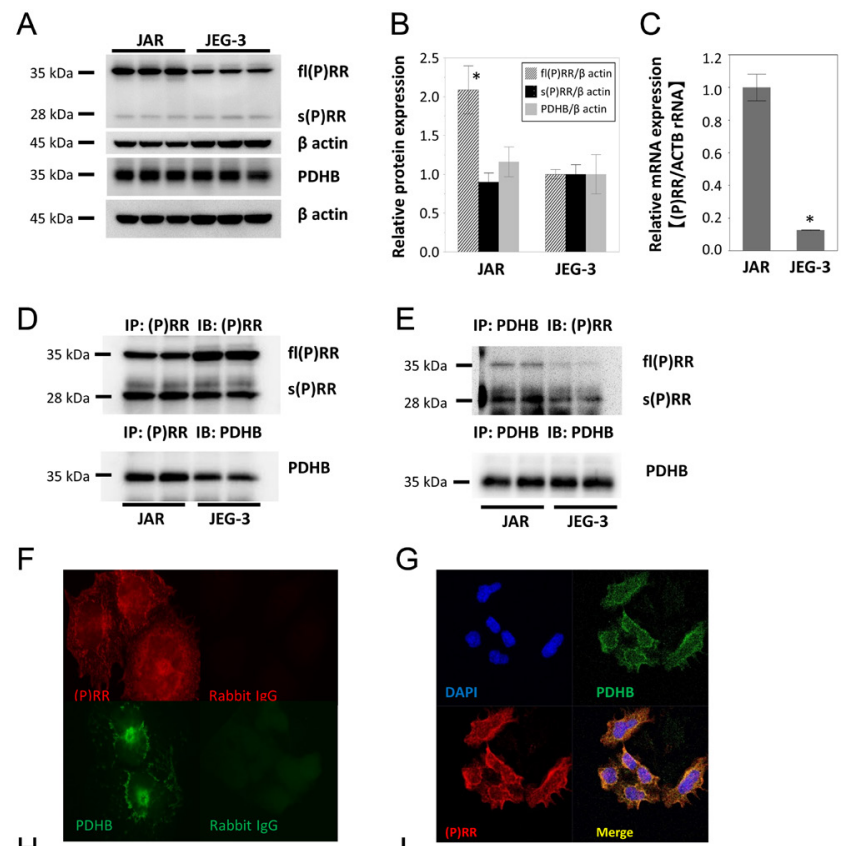

$\mathrm{H}$

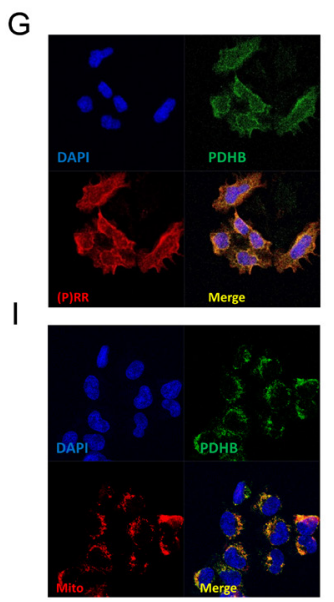

\section{Figure 1}

Expression and interaction of (P)RR and PDHB were confirmed in two trophoblast cell lines, JAR and JEG-3. (A) Immunoblots showing the protein expression of (P)RR and PDHB in two trophoblast cell lines. (B) Densitometry results show that JAR cells expressed more full-length (P)RR than JEG-3 cells $(n=6)$. (C) JAR cells expressed more (P)RR mRNA than JEG-3 cells $(n=6)$. (D) PDHB co-immunoprecipated with (P)RR in JAR and JEG-3 cells. (E) Predominantly s(P)RR and less fl(P)RR co-immunoprecipited with PDHB. (F) Rabbit IgG as negative control showed negligible fluorescence signal in JAR cells. (G) (P)RR (red) and PDHB (green) co-localized in the cytoplasm. (H) (P)RR (green) co-localized with mitochondrial marker (red). (I) PDHB co-localized with mitochondrial marker (red). IP, immunoprecipitation; IB, immunoblot; fI(P)RR, full-length (P)RR; s(P)RR, soluble (P)RR concentration. Measurements were performed according to the manufacturer's instructions using the SpectraMax i3 microplate reader (Molecular Devices Japan) in duplicates and media as control. Intra- and inter-assay coefficients of variation by the root mean square method were 1.31 and $2.54 \%, 2.30$ and $3.76 \%$, and 2.03 and $4.77 \%$ for PDH activity, pyruvate assay, and acetyl CoA assay, respectively.

\section{Statistical analysis}

All tests were performed using JMP Pro software, version 13.0.0 (SAS Institute Inc) and SPSS Statics 21 (IBM). Summary data are presented means \pm S.D. Statistical significance was analyzed using one-way ANOVA followed by the Tukey-Kramer post hoc test or Wilcoxon's pair-wise non-parametric test. Statistical significance for trend was analyzed by Jonckheere-Terpstra test using EZR (Saitama Medical Center, Jichi Medical University, Saitama, Japan), which is a graphical user interface for R (The R Foundation for Statistical Computing, Vienna, Austria). A $P$ value of $<0.05$ was considered statistically significant.

\section{Results}

\section{Co-expression of (P)RR and PDHB in human trophoblast cells}

The protein expressions of (P)RR and PDHB in JAR and JEG-3 cells were examined by Western blot (Fig. 1A). JAR cells expressed 2.1-fold full-length (P)RR protein than JEG-3 cells, but protein levels of $s(P) R R$ and PDHB were not significantly different (Fig. 1B). JAR cells expressed significantly more (P)RR mRNA compared to JEG-3 cells (Fig. 1C). Protein-protein interaction between (P)RR and PDHB was revealed by immunoprecipitation (Fig. 1D and E), and the type of PRR protein that co-immunoprecipitated with PDHB was predominantly $s(P) R R$ with some fulllength (P)RR (Fig. 1E). Because of the greater expression of (P)RR in JAR cells compared to JEG-3 cells, we used JAR cells in the subsequent experiments. Co-expression of (P)RR and PDHB was observed in immunofluorescence studies (Fig. 1F and G). Staining by Mitotracker agent revealed that $s(\mathrm{P}) \mathrm{RR}$ and $\mathrm{PDHB}$ are co-expressed in the mitochondria (Fig. 1H and I).

\section{Effects of hypoxia on (P)RR expression and PDHB function in JAR cells}

Hypoxia treatment $\left(1 \% \mathrm{O}_{2}\right)$ using a chamber significantly increased the intracellular expression of $s(\mathrm{P}) \mathrm{RR}$ protein by 
approximately 2 -fold ( $21 \%$ vs $1 \% \mathrm{O}_{2}$ ), and the increase in $\mathrm{s}(\mathrm{P}) \mathrm{RR}$ depended on the degree of hypoxia (Fig. $2 \mathrm{~A}$ and $B)$. The difference in $s(P) R R$ protein level was significant between 8 and $1 \% \mathrm{O}_{2}(P=0.005)$ but not between 5 and $1 \% \mathrm{O}_{2} \quad(P=0.20)$. The $\mathrm{fl}(\mathrm{P}) \mathrm{RR}$ protein decreased with hypoxia (Fig. 2A and $\mathrm{B}$ ). The increase in $\mathrm{s}(\mathrm{P}) \mathrm{RR}$ and decrease in $\mathrm{fl}(\mathrm{P}) \mathrm{RR}$ in response to hypoxia were also time dependent (Fig. 2C and D). Hypoxia treatment did not significantly alter (P)RR mRNA (Fig. 2C) or PDHB protein levels (Fig. 2A, B, C and D). In contrast to the increase in intracellular $\mathrm{s}(\mathrm{P}) \mathrm{RR}$, hypoxia treatment decreased $\mathrm{s}(\mathrm{P})$ RR released into the culture medium (Fig. 2F). Chamber treatment with normoxic air as control did not alter $\mathrm{s}(\mathrm{P})$ RR protein level (Supplementary Fig. 1, see section on supplementary materials given at the end of this article), and in JEG-3 cells, hypoxia treatment had no significant effect on intracellular (P)RR expression (Supplementary
Fig. 2A and B). In JAR cells, chemical hypoxia by cobalt chloride increased intracellular s(P)RR and decreased $\mathrm{fl}(\mathrm{P})$ $\mathrm{RR}$, similar to the results obtained by hypoxia treatment using a chamber (Supplementary Fig. 3A and B).

\section{Effects of (P)RR knockdown on PDHB expression and activity during hypoxia}

To investigate the role of $(\mathrm{P}) \mathrm{RR}$ during hypoxia, we carried out knockdown experiments by siRNA. (P)RR siRNA suppressed (P)RR mRNA expression by 95\% (Fig. 3A) and full-length and soluble (P)RR protein levels by 42 and $40 \%$, respectively (Fig. 3B, C and D), compared to negative control siRNA. In contrast to the controls cells where PDHB expression was maintained after hypoxia (Fig. 2A, B, C and D), (P)RR siRNA-treated cells showed significant decreases in PDHB protein expression (Fig. 3B and E,
A

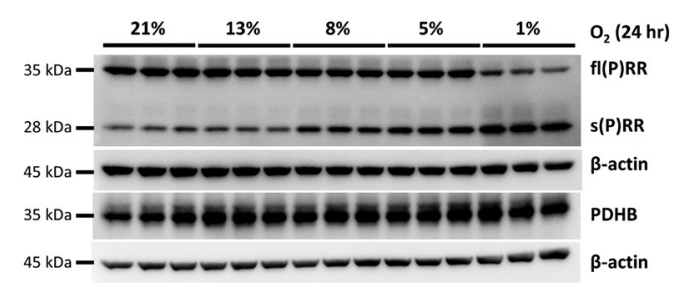

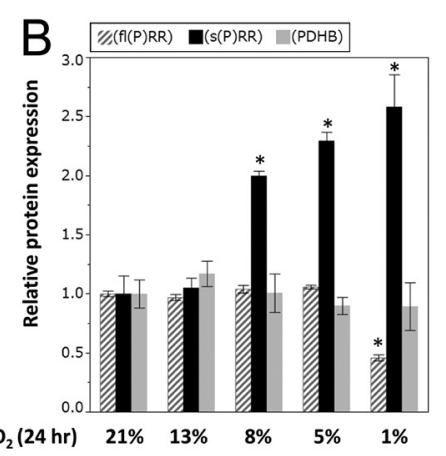

C
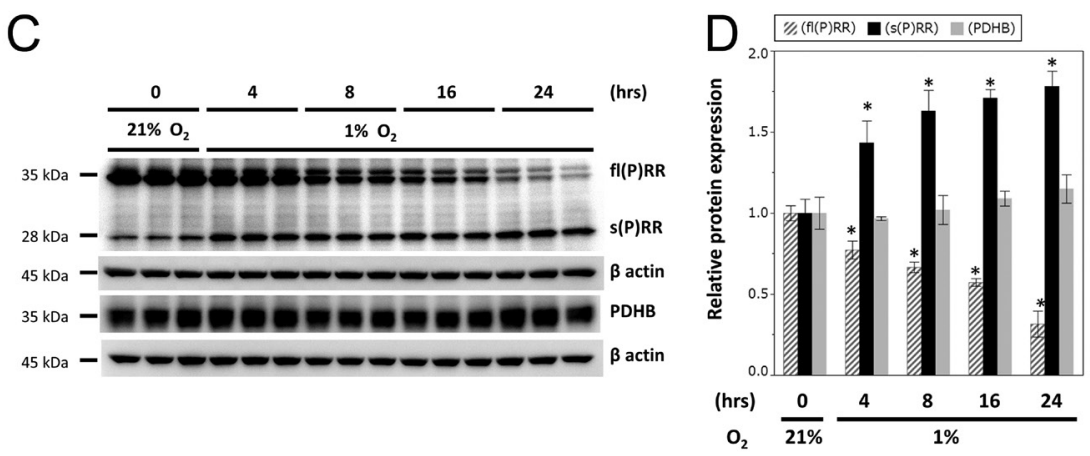

$E$

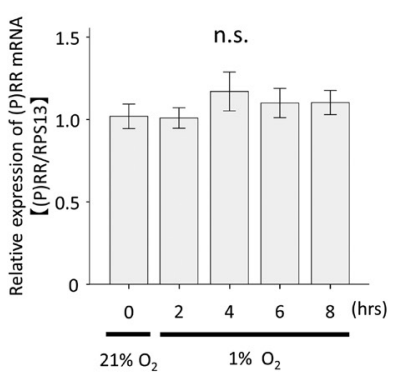

$\mathrm{F}$

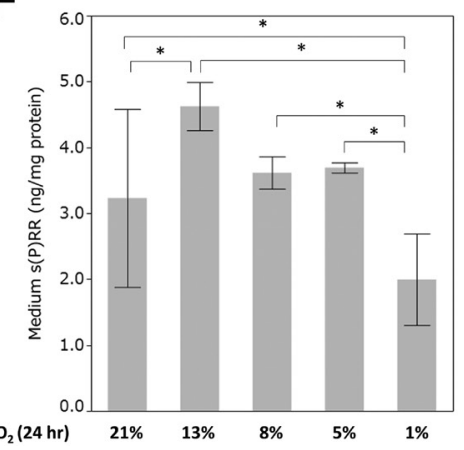

Figure 2

Hypoxia dose- and time-dependently increased intracellular S(P)RR in JAR cells. (A) Representative immunoblot for dose-response of fl(P)RR, S(P)RR, and $P D H B$. The increase in intracellular $S(P) R R$ was dependent on the degree of hypoxia. (B) Hypoxia dose-dependently increased S(P)RR and decreased fl(P)RR, while PDHB was unchanged ( $n=3$ cultures/group). (C) Representative immunoblot for time-course of $\mathrm{fl}(\mathrm{P}) \mathrm{RR}, \mathrm{s}(\mathrm{P}) \mathrm{RR}$, and PDHB response to hypoxia. (D) Hypoxia ( $1 \% \mathrm{O}_{2}$ chamber) increased $s(P) R R$ and decreased $f(P) R R$, but there was no significant change in the PDHB protein level ( $n=3$ cultures/group). (E) (P)RR mRNA expression was not significantly altered by hypoxia. (F) Secreted s(P)RR in the culture medium was decreased with hypoxia ( $n=6$ cultures/group). ${ }^{\star} P<0.05$ vs $21 \% \mathrm{O}_{2}$ control; n.s., not significant. 
$56 \%$ reduction in siRNA-treated cells) and activity (Fig. 3F, 37\% reduction in siRNA-treated cells) after hypoxia treatment.

\section{PDH-associated metabolites and PDHB phosphorylation with (P)RR siRNA}

Next, we measured the concentrations of metabolites affected by PDH activity. The PDH complex contributes to the transformation of pyruvate into acetyl-CoA. Intracellular pyruvate concentration increased after hypoxic treatment, and this pattern was similar between cells treated by control siRNA and (P)RR siRNA (Fig. 4A). In contrast, intracellular acetyl-CoA concentration was not affected by hypoxia but was decreased in siRNAtransfected cells compared to control cells (Fig. 4B). Because tyrosine phosphorylation of $\mathrm{PDHB}$ is known
A

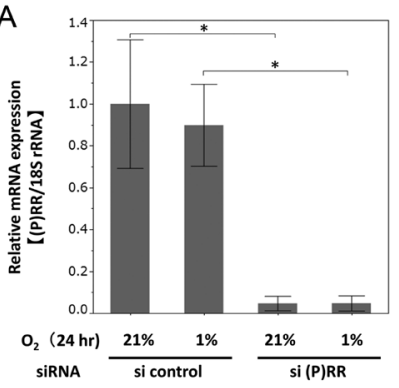

C

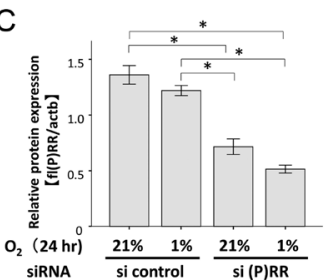

E

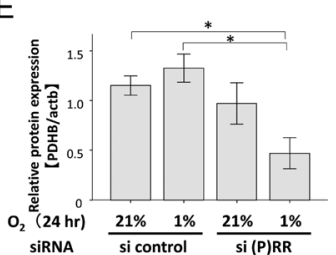

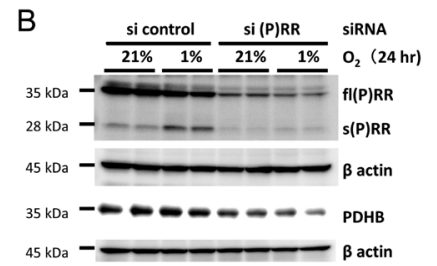

$\mathrm{D}$

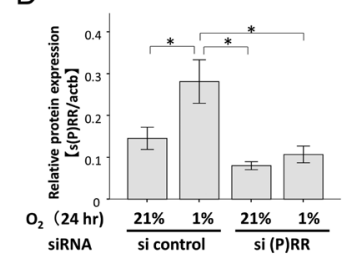

F

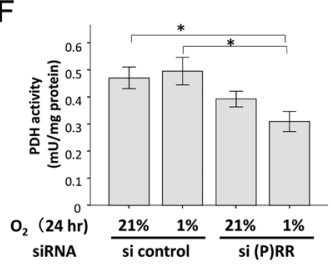

Figure 3

(P)RR knockdown by siRNA uncovered hypoxia-induced reduction in PDHB expression and PDH activity. The JAR cells were treated with (P)RR or control siRNA for $72 \mathrm{~h}$ and subjected to 1 or $21 \% \mathrm{O}_{2}$ for $24 \mathrm{~h}$. (A) (P)RR siRNA decreased (P)RR mRNA expression ( $n=4$ cultures/group). (B) Representative immunoblot with $\beta$ actin for loading control. (C) (P)RR siRNA treatment reduced fl(P)RR to approximately 50\% ( $n=4$ cultures/ group). (D) S(P)RR was suppressed approximately $50 \%$ by (P)RR siRNA ( $n=4$ cultures/group). (E) PDHB protein expression decreased with (P)RR siRNA during hypoxia ( $n=4$ cultures/group). (F) PDH activity decreased $36 \%$ with (P)RR siRNA compared to $1 \% \mathrm{O}_{2}$ control ( $n=8$ cultures/group). ${ }^{*} P<0.05$; ANOVA with Tukey-Kramer test. to lead to ubiquitination and degradation of the $\mathrm{PDH}$ complex, we assessed the phosphorylation status of PDHB. Immunoblotting study with anti p-tyrosine against immunoprecipitated $\mathrm{PDHB}$ protein revealed that phospholylation levels of PDHB was increased by (P)RR siRNA treatment (Fig. 4C and D), suggesting that hypoxia-induced $\mathrm{s}(\mathrm{P}) \mathrm{RR}$ binds to $\mathrm{PDHB}$ to prevent tyrosine phosphorylation of $\mathrm{PDHB}$, thereby preserving PDHB protein level and the activity of $\mathrm{PDH}$ complex in trophoblasts.

\section{(P)RR processing enzyme inhibition and (P)RR and PDHB expression during hypoxia}

Since (P)RR is cleaved by various enzymes to generate $s(P) R R$ with subtle differences in terminal amino acid sequence, effects of (P)RR processing enzyme inhibitors on the protein expression of $\mathrm{fl}(\mathrm{P}) \mathrm{RR}, \mathrm{s}(\mathrm{P}) \mathrm{RR}$, and $\mathrm{PDHB}$ were determined. Inhibitors of furin (Furin inhibitor II, Fig. 5A and B) and site 1 protease (PF429242, Fig. 5C and D) decreased $s(P) R R$ and increased $f(P) R R$, suggesting the involvement of these enzymes in the processing of $(\mathrm{P})$ RR in trophoblasts during hypoxia. Meanwhile, inhibitor of ADAM19 (GM6001, Fig. 5E and F) did not have significant effect on the protein expression of $\mathrm{fl}(\mathrm{P}) \mathrm{RR}, \mathrm{s}(\mathrm{P})$ $\mathrm{RR}$, and PDHB during hypoxia. Only with site 1 protease inhibitor, PF429242, the PDHB protein expression showed a significant trend to decrease with inhibitor treatment (Fig. 5C and D).

\section{Discussion}

We examined the possible molecular basis of the association between $\mathrm{s}(\mathrm{P}) \mathrm{RR}$, gestational hypertension/ preeclampsia, and sustained fetal growth by determining the response of (P)RR to hypoxia in trophoblasts. This report showed for the first time that intracellular $s(P) R R$ is increased by hypoxia and interacts with PDHB to stabilize PDHB protein and PDH activity in trophoblast cells. These findings support our hypothesis that increase in $s(P) R R$ may be involved in sustained fetal growth in the face of decreased placental perfusion.

Our group recently reported that hypoxia increases (P)RR protein in cultured adipocytes without altering its mRNA level (Seki et al. 2018). Our present study showed that hypoxia alters (P)RR protein also in trophoblasts. (P) $\mathrm{RR}$ protein is known to be increased by stimuli such as nutrient starvation (Mizuguchi et al. 2018), high glucose (Li \& Siragy 2015), and angiotensin II (Li et al. 2015) in various cell types. In contrast to other stimuli that 
A

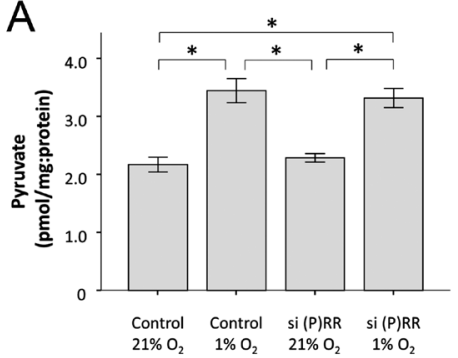

C

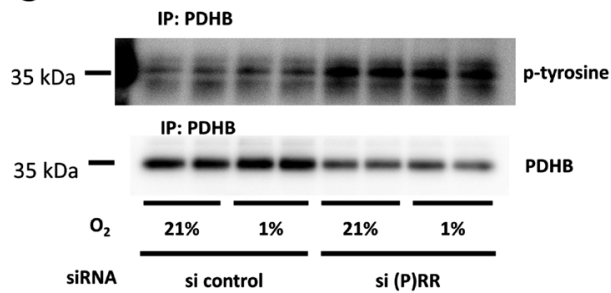

B
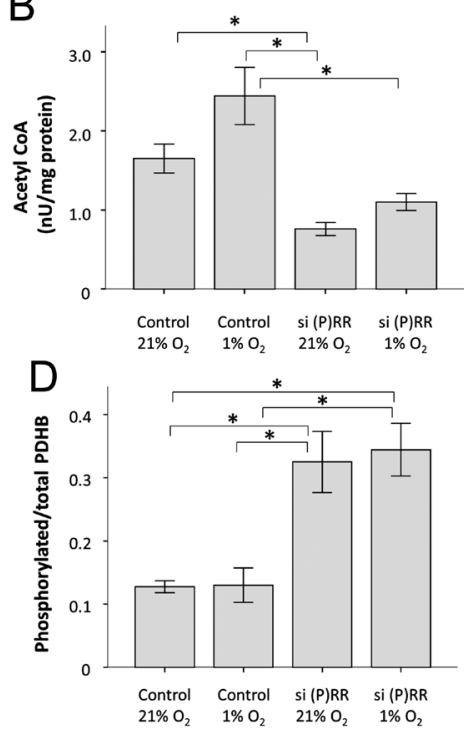

\section{Figure 4}

$\mathrm{PDH}$-associated metabolites were altered and PDHB phosphorylation increased with (P)RR siRNA treatment in JAR cells. (A) Hypoxia treatment for $24 \mathrm{~h}$ increased intracellular pyruvate concentration, and this pattern was not altered by (P)RR siRNA treatment ( $n=8$ cultures/ group). (B) (P)RR siRNA decreased acetyl CoA in both normoxic and hyposic trophoblasts $(n=8$ cultures/group). (C) Phosphorylated PDHB increased with (P)RR siRNA treatment. (D) The increase in phosphorylated PDHB by (P)RR siRNA was approximately 3 -fold in both normoxic and hypoxic trophoblasts ( $n=4$ cultures/group). $* P<0.05$; ANOVA with Tukey-Kramer test. increased (P)RR at its mRNA level, hypoxia increased (P) RR protein without altering its mRNA level in adipocytes in the study by Seki et al. and hypoxia specifically increased intracellular $s(\mathrm{P}) \mathrm{RR}$ in the trophoblasts in our study. It needs to be kept in mind that physiological $\mathrm{O}_{2}$ concentration is 5 to $8 \%$ in the placenta, and so comparing $21-1 \% \mathrm{O}_{2}$ may be experimental, and the difference in $\mathrm{s}(\mathrm{P})$ RR between 8 and $1 \% \mathrm{O}_{2}$ was significant but smaller than that observed between 21 and $1 \% \mathrm{O}_{2}$.

Modulation of (P)RR protein formation/degradation, (P)RR cleavage, and/or intracellular distribution may be involved in the hypoxia-induced increase of intracellular $\mathrm{s}(\mathrm{P}) \mathrm{RR}$, and its mechanism should be explored in further research.

Another major finding of this study was that (P)RR and PDHB were co-expressed and interacted with each other in trophoblasts. Kanda et al. showed direct binding of PDHB protein and (P)RR at its N-terminal domain using yeast two-hybrid and co-immunoprecipitation experiments. The study showed that (P)RR binding protects PDHB from tyrosine phosphorylation, therefore preventing ubiquitination and degradation of PDHB (Kanda et al. 2015). The N-terminal domain of (P)RR comprises $\mathrm{s}(\mathrm{P}) \mathrm{RR}$, and our finding that predominantly $\mathrm{s}(\mathrm{P})$ RR instead of full-length (P)RR co-immunoprecipitated with PDHB is consistent with implications of the findings by Kanda et al.

Further, we probed into the role of increased intracellular $\mathrm{s}(\mathrm{P}) \mathrm{RR}$ during hypoxia in trophoblasts. Generally, hypoxia decreases PDH activity via induction of HIF-1, which increases pyruvate dehydrogenase kinase 1 (PDK1) that phosphorylates PDH E1 alpha subunit and inactivates the PDH enzyme complex (Kim et al. 2006). Hypoxic repression of PDH activity is considered a metabolic adaptation to hypoxia and has been shown in various cells types, such as pancreatic (Golias et al. 2016) and hepatocellular carcinoma cells (Zimmer et al. 2016). In our study, PDH activity was maintained under hypoxia in trophoblasts, and (P)RR siRNA treatment uncovered the hypoxic suppression of PDH activity. As (P)RR knockdown significantly increased PDHB phosphorylation, our results suggested that hypoxic induction of $s(\mathrm{P}) \mathrm{RR}$ inhibited PDHB phosphorylation to maintain its protein level and PDH activity. This was also supported by decreased acetylCoA levels in (P)RR siRNA-treated cells.

In addition, we showed that site 1 protease and furin were the enzymes most likely to cleave fl(P)RR to produce $s(\mathrm{P}) \mathrm{RR}$ in trophoblasts during hypoxia. It is postulated that subtly different $s(\mathrm{P}) \mathrm{RR}$ produced by different cleaving enzymes exhibit disparate intra- and para-cellular distribution and function (Nakagawa et al. 2017). It would be of interest in future studies to test if $s(P) R R s$ that differ by only a few amino acids would exert differential effects on the function of trophoblasts.

In a clinical context, the finding that hypoxia rather decreased $s(\mathrm{P}) \mathrm{RR}$ concentration in the culture media is of interest. In preeclampsia, plasma s(P)RR may be increased not because the secretion of $s(P) R R$ is increased, but as a reflection of tissue damage; intracellular $s(\mathrm{P}) \mathrm{RR}$ increased in response to hypoxia may spill out into blood flow from damaged cells. S(P)RR concentration in umbilical cord plasma (Watanabe et al. 2013) is higher than mean maternal plasma $\mathrm{s}(\mathrm{P}) \mathrm{RR}$ concentration at delivery (Watanabe et al. 2012), and plasma s(P)RR concentration 
B

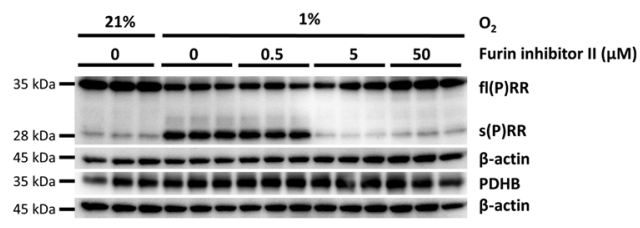

C

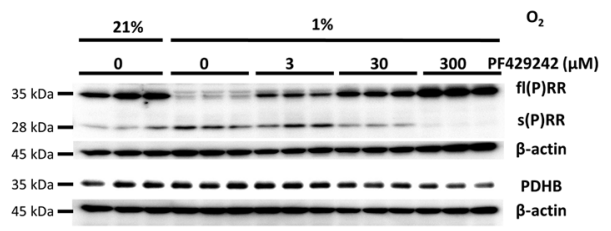

Furin inhibitor II $(\mu \mathrm{M})$

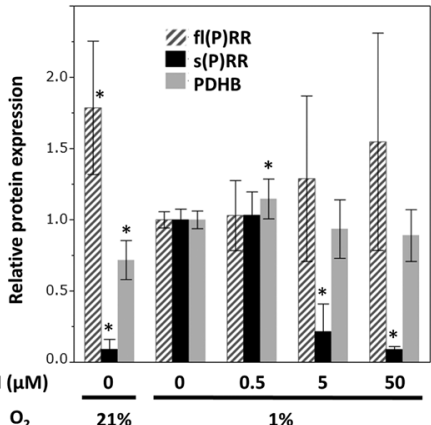

$\mathrm{O}_{2}$
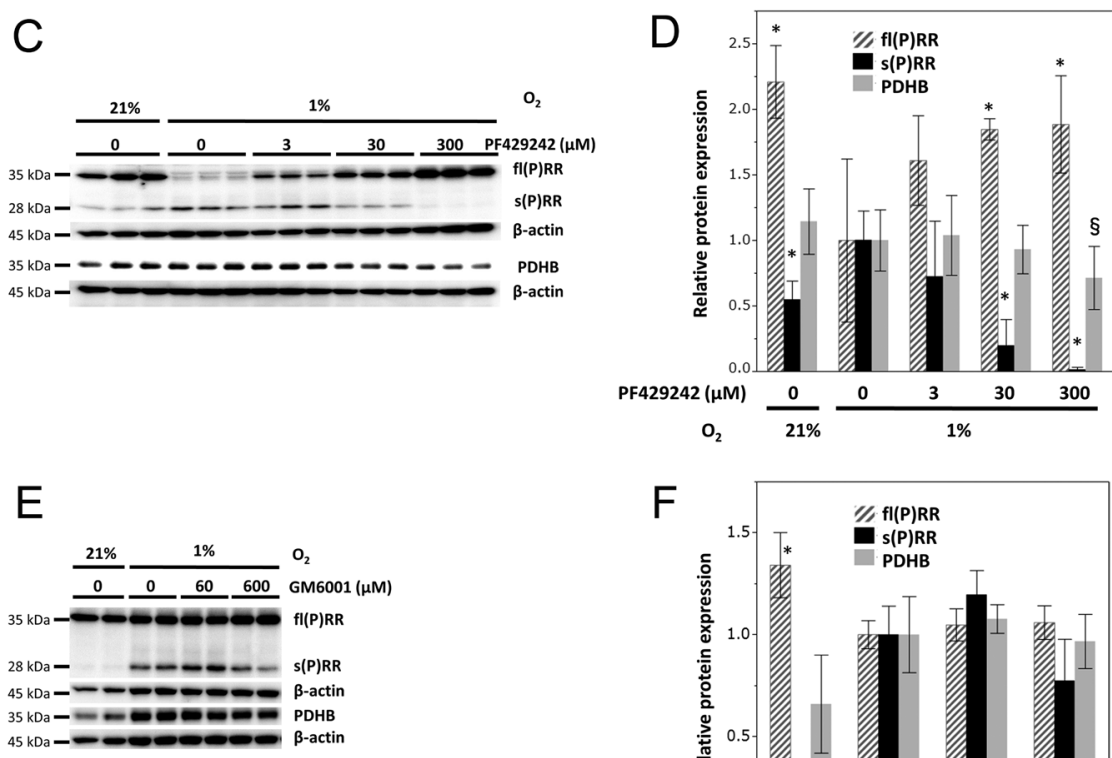

$\mathrm{F}$

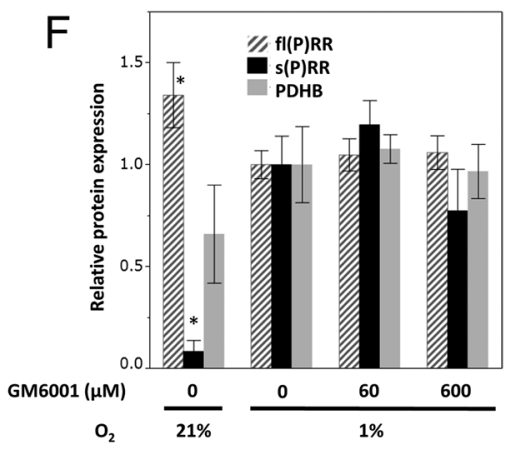

\section{Figure 5}

Furin and site 1 protease inhibitors suppressed s(P)RR generation during hypoxia. The JAR cells were subjected to hypoxia and/or inhibitors for 24 h. (A) Furin inhibitor II dose-dependently decreased $s(P) R R$ and increased fl(P)RR. (B) S(P)RR expression reduced to approximately $10 \%$ with furin inhibitor II ( $n=6$ cultures/group). (C) Site 1 protease inhibitor, PF429242, reduced s(P)RR and increased fl(P)RR. (D) S(P)RR expression reduced to approximately $2 \%$ with PF429242 $(n=7$ cultures/group). (E) ADAM-19 inhibitor, GM6001, did not significantly alter $s(P) R R$ expression in trophoblasts during hypoxia. (F) Graphical summary of $n=6$ cultures/group data using GM6001. $* P<0.05$ vs $1 \% \mathrm{O}_{2}, 0 \mu \mathrm{M}$ control, Wilcoxon's pair-wise non-parametric test; ${ }^{\S} P<0.05$ for trend by Jonckheere-Terpstra test. at early pregnancy predicts subsequent blood pressure elevation (Watanabe et al. 2012). Although hypoxia did not directly increase secreted $s(P) R R$ from trophoblasts, possibility remains that elevated maternal $s(P) R R$ concentration originates from the placenta or fetus and reflects placental ischemia.

The significance of hypoxic induction of $s(\mathrm{P}) \mathrm{RR}$ may of interest also from the context of syncytialization. Previous study has shown that both ATP production and oxygen consumption are increased during syncytialization (Maloyan et al. 2012), and downregulation of PDK4, which inactivates the PDH complex, is demonstrated to be critical for the switch of carbohydrate catabolism from glycolysis to oxidative phosphorylation during syncytialisation. Increased $\mathrm{s}(\mathrm{P}) \mathrm{RR}$ during hypoxia may support PDH activity and maintain syncytialisation, which is necessary for proper placental formation and function.

Currently, effects of elevated s(P)RR on the pregnant mother is not clear. Renal tubular intraluminal $\mathrm{s}(\mathrm{P})$
RR has been demonstrated to possess renin catalytic activity, augmenting the formation of angiotensin I from angiotensinogen (Gonzalez et al. 2011). Circulating s(P)RR likely exhibits renin catalytic activity when bound with prorenin. Prorenin is the inactive precursor of renin, and prorenin circulates in blood at concentrations that are ten times higher, or in diabetes and pregnancy up to 100 times higher, than that of active renin (Krop et al. 2013). Increase in circulating $\mathrm{s}(\mathrm{P}) \mathrm{RR}$ may activate systemic reninangiotensin system. On the other hand, because $\mathrm{s}(\mathrm{P}) \mathrm{RR}$ is not a membrane-bound receptor, $\mathrm{s}(\mathrm{P}) \mathrm{RR}$ binding with prorenin would not be followed by cellular events such as MAPK/ Erk activation or nuclear translocation of promyelocytic leukemia zinc finger activating phosphoinositide 3-kinase, and this way, it is also postulated that circulating $s(P) R R$ may buffer some of the effects of prorenin on cellular events. Delineations of the consequences of $s(P) R R$ induction await further research.

It needs to be kept in mind that our study was performed using cultured human choriocarcinoma cell 
lines, and some of the findings may not truly reflect physiological responses of native trophoblast cells. Also, the state of preeclampsia, placental insufficiency, or hypoxia may alter an array of systemic and local factors, and involvement of related parameters other than hypoxia should also be noted.

In conclusion, we demonstrated for the first time that increased intracellular $s(P) R R$ in response to hypoxia supports PDH function in trophoblasts, which may play a pivotal role in the maintenance of placental normality. (P)RR or its soluble form may be a potential therapeutic target for hypertensive disorders of pregnancy.

\section{Supplementary materials}

This is linked to the online version of the paper at https://doi.org/10.1530/ JME-19-0050.

\section{Declaration of interest}

The authors declare that there is no conflict of interest that could be perceived as prejudicing the impartiality of the research reported.

\section{Funding}

This study was supported in part by a JSPS KAKENHI Grant Number $16 \mathrm{H} 05316$ to $\mathrm{A}$ I and a JSPS KAKENHI Grant Number $19 \mathrm{~K} 08736$ to M Y.

\section{Acknowledgments}

The authors thank Dr Katsuhiko Naruse for valuable advice on cell selection and experimental methods.

\section{References}

Amari Y, Morimoto S, Nakajima F, Ando T \& Ichihara A 2016 Serum soluble (pro)renin receptor levels in maintenance hemodialysis patients. PLOS ONE 11 e0158068. (https://doi.org/10.1371/journal. pone.0158068)

Fukushima A, Kinugawa S, Homma T, Masaki Y, Furihata T, Abe T, Suga T, Takada S, Kadoguchi T, Okita K, et al. 2013 Increased plasma soluble (pro)renin receptor levels are correlated with renal dysfunction in patients with heart failure. International Journal of Cardiology 168 4313-4314. (https://doi.org/10.1016/j. ijcard.2013.04.176)

Golias T, Papandreou I, Sun R, Kumar B, Brown NV, Swanson BJ, Pai R, Jaitin D, Le QT, Teknos TN,et al. 2016 Hypoxic repression of pyruvate dehydrogenase activity is necessary for metabolic reprogramming and growth of model tumours. Scientific Reports $\mathbf{6}$ 31146. (https://doi.org/10.1038/srep31146)

Gonzalez AA, Lara LS, Luffman C, Seth DM \& Prieto MC 2011 Soluble form of the (pro)renin receptor is augmented in the collecting duct and urine of chronic angiotensin II-dependent hypertensive rats. Hypertension 57 859-864. (https://doi.org/10.1161/ HYPERTENSIONAHA.110.167957)

Hamada K, Taniguchi Y, Shimamura Y, Inoue K, Ogata K, Ishihara M, Horino T, Fujimoto S, Ohguro T, Yoshimoto Y,et al. 2013 Serum level of soluble (pro)renin receptor is modulated in chronic kidney disease. Clinical and Experimental Nephrology 17 848-856. (https://doi. org/10.1007/s10157-013-0803-y)

Ichihara A \& Yatabe MS 2019 The (pro)renin receptor in health and disease. Nature Reviews: Nephrology 15 693-712. (https://doi. org/10.1038/s41581-019-0160-5)

Ichihara A, Sakoda M, Kurauchi-Mito A, Narita T, Kinouchi K, Murohashi-Bokuda K \& Itoh H 2010 Possible roles of human (pro) renin receptor suggested by recent clinical and experimental findings. Hypertension Research 33 177-180. (https://doi.org/10.1038/ hr.2009.214)

Kanda A, Noda K \& Ishida S 2015 ATP6AP2/(pro)renin receptor contributes to glucose metabolism via stabilizing the pyruvate dehydrogenase E1 beta subunit. Journal of Biological Chemistry 290 9690-9700. (https://doi.org/10.1074/jbc.M114.626713)

Kim JW, Tchernyshyov I, Semenza GL \& Dang CV 2006 HIF-1-mediated expression of pyruvate dehydrogenase kinase: a metabolic switch required for cellular adaptation to hypoxia. Cell Metabolism 3 177-185. (https://doi.org/10.1016/j.cmet.2006.02.002)

Kimura S, Morimoto S, Yamashita K, Takano N, Mizuguchi Y, Niiyama M, Bokuda K, Yatabe M, Yatabe J, Watanabe D,et al. 2016. PS 02-47 relationships between serum soluble (pro)renin receptor levels and obesity- or metabolism-related factors in essential hypertensive patients. Journal of Hypertension 34 e116-e117.

Kinouchi K, Ichihara A, Sano M, Sun-Wada GH, Wada Y, Ochi H, Fukuda T, Bokuda K, Kurosawa H, Yoshida N,et al. 2013 The role of individual domains and the significance of shedding of ATP6AP2/ (pro)renin receptor in vacuolar $\mathrm{H}(+)$-ATPase biogenesis. PLOS ONE 8 e78603. (https://doi.org/10.1371/journal.pone.0078603)

Krop M, Lu X, Danser AH \& Meima ME 2013 The (pro)renin receptor. A decade of research: what have we learned? Pflugers Archiv 465 87-97. (https://doi.org/10.1007/s00424-012-1105-z)

Li C \& Siragy HM 2015 (Pro)renin receptor regulates autophagy and apoptosis in podocytes exposed to high glucose. American Journal of Physiology: Endocrinology and Metabolism 309 E302-E310. (https://doi. org/10.1152/ajpendo.00603.2014)

Li W, Liu J, Hammond SL, Tjalkens RB, Saifudeen Z \& Feng Y 2015 Angiotensin II regulates brain (pro)renin receptor expression through activation of cAMP response element-binding protein. American Journal of Physiology: Regulatory, Integrative and Comparative Physiology 309 R138-R147. (https://doi.org/10.1152/ajpregu.00319.2014)

Lu X, Wang F, Xu C, Soodvilai S, Peng K, Su J, Zhao L, Yang KT, Feng Y, Zhou SF,et al. 2016 Soluble (pro)renin receptor via beta-catenin enhances urine concentration capability as a target of liver $\mathrm{X}$ receptor. PNAS 113 E1898-E1906. (https:/doi.org/10.1073/ pnas.1602397113)

Maloyan A, Mele J, Muralimanohara B \& Myatt L 2012 Measurement of mitochondrial respiration in trophoblast culture. Placenta 33 456-458. (https://doi.org/10.1016/j.placenta.2012.01.016)

Mizuguchi Y, Morimoto S, Kimura S, Takano N, Yamashita K, Seki Y, Bokuda K, Yatabe M, Yatabe J, Watanabe D,et al. 2018 Prediction of response to medical therapy by serum soluble (pro)renin receptor levels in Graves' disease. PLOS ONE 13 e0195464. (https://doi. org/10.1371/journal.pone.0195464)

Morimoto S, Ando T, Niiyama M, Seki Y, Yoshida N, Watanabe D, Kawakami-Mori F, Kobori H, Nishiyama A \& Ichihara A 2014 Serum soluble (pro)renin receptor levels in patients with essential hypertension. Hypertension Research 37 642-648. (https://doi. org/10.1038/hr.2014.46)

Nakagawa T, Suzuki-Nakagawa C, Watanabe A, Asami E, Matsumoto M, Nakano M, Ebihara A, Uddin MN \& Suzuki F 2017 Site- 1 protease is required for the generation of soluble (pro)renin receptor. Journal of Biochemistry 161 369-379. (https://doi. org/10.1093/jb/mvw080)

Narita T, Ichihara A, Matsuoka K, Takai Y, Bokuda K, Morimoto S, Itoh H \& Seki H 2016 Placental (pro)renin receptor expression and plasma 
soluble (pro)renin receptor levels in preeclampsia. Placenta 37 72-78. (https://doi.org/10.1016/j.placenta.2015.11.007)

Nguyen G, Delarue F, Burckle C, Bouzhir L, Giller T \& Sraer JD 2002 Pivotal role of the renin/prorenin receptor in angiotensin II production and cellular responses to renin. Journal of Clinical Investigation 109 1417-1427. (https://doi.org/10.1172/JCI14276)

Seki Y, Yatabe M, Suda C, Morimoto S \& Ichihara A 2018 Elevated (pro) renin receptor expression contributes to maintaining aerobic metabolism in growth hormone deficiency. Journal of the Endocrine Society 2 252-265. (https://doi.org/10.1210/js.2017-00447)

Watanabe N, Bokuda K, Fujiwara T, Suzuki T, Mito A, Morimoto S, Jwa SC, Egawa M, Arai Y, Suzuki F,et al. 2012 Soluble (pro)renin receptor and blood pressure during pregnancy: a prospective cohort study. Hypertension 60 1250-1256. (https://doi.org/10.1161/ HYPERTENSIONAHA.112.197418)

Watanabe N, Morimoto S, Fujiwara T, Suzuki T, Taniguchi K, Ando T, Kimura T, Sago H \& Ichihara A 2013 Association between soluble (pro)renin receptor concentration in cord blood and small for gestational age birth: a cross-sectional study. PLOS ONE 8 e60036. (https://doi.org/10.1371/journal.pone.0060036)

Zimmer AD, Walbrecq G, Kozar I, Behrmann I \& Haan C 2016 Phosphorylation of the pyruvate dehydrogenase complex precedes HIF-1-mediated effects and pyruvate dehydrogenase kinase 1 upregulation during the first hours of hypoxic treatment in hepatocellular carcinoma cells. Hypoxia 4 135-145. (https://doi. org/10.2147/HP.S99044)

Received in final form 2 January 2020

Accepted 17 January 2020

Accepted Manuscript published online 17 January 2020
(C) 2020 Society for Endocrinology Published by Bioscientifica Ltd. Printed in Great Britain 